\title{
Lineshape analysis of stimulated Raman spectra of the near-nozzle region of free jet expansions
}

\author{
A. Ramos *, J. Santos, L. Abad ${ }^{1}$, D. Bermejo, V. J. Herrero, and I. Tanarro \\ Instituto de Estructura de la Materia, CSIC, Serrano 121, 28006 Madrid, Spain and \\ ${ }^{1}$ Departamento de Física, Universidad Alfonso X el Sabio, Villanueva de la Cañada, 28691 Madrid, Spain
}

(Dated: Published online 20 April 2009)

\begin{abstract}
An anomalous lineshape of stimulated Raman spectra obtained from the region very close to the nozzle of supersonic pulsed expansions of nitrogen is presented. High resolution Raman spectra of the $Q$ branch of the fundamental vibration mode of $\mathrm{N}_{2}$ have been recorded from two different nitrogen expansions at $T_{0}=295 \mathrm{~K}$ and $P_{0}=1.5-3.5 \mathrm{bar}$, the lasers crossing the jet axis in the range $z / D=0.25-1.25$, where $D$ is the effective nozzle diameter. The combination of Doppler shifts and strong gradients of density and temperature in the near-nozzle region yield an inhomogeneous broadening and a double-peak structure of the recorded Raman line profiles. The comparation of the experimental results with the simulation of the Raman spectrum from this region provides valuable information about the near-nozzle flow field. The lineshape described here is different from another reported previously in the literature, which is based on a depletion of the density of free molecules on the axis due to condensation.
\end{abstract}

\section{INTRODUCTION}

Free jet expansions, with their combination of fast cooling and rarefaction, provide a unique environment for studies of low temperature energy transfer, chemical reactivity, and agregation processes in gases. Simple jet models assuming an isentropic expansion have been of great help for the rationalization of their physical properties and have often proven strikingly accurate for the prediction of jet characteristics, specially in the centerline of axisymmetric expansions, beyond a certain distance to the origin $[1,2]$. In the immediate vicinity of the jet source nozzle, the validity of the current simple jet models is questionable and should be assessed with experimental measurements.

Optical spectroscopies are a common tool for studying supersonic free jet expansions of neutral gases and plasma sources, as they do not practically disturb the medium. Among them, high resolution spectroscopic techniques, like visible absorption[3], diode-laser infrared absorption $[4,5]$, Fourier transform infrared (FTIR) absoption spectroscopy $[6,7]$, Fourier transform microwave (FTMW) measurements [8], resonance enhanced multiphoton ionization (REMPI) [9, 10], cavity ring down spectroscopy [11], or nonlinear Raman spectroscopies (SRS, CARS,...) $[12,13]$, have been widely used to measure vibrationrotation spectra of molecules cooled in the expansion and for the analysis of phenomena naturally occurring, or externally induced, in free jets and supersonic beams, like nonequilibrium between the molecular degrees of freedom $[9,10]$, nucleation and clustering of the gas $[11,12]$, photodisociation and photon induced reactions [14, 15], or interactions of molecules with fields [16].

In the spectra obtained from jets with techniques that have a great field depth, like infrared absorption, the

\footnotetext{
*E-mail: a.ramos@iem.cfmac.csic.es
}

measured intensity and the profile of the spectral lines are the result of an integration along the line of sight, containing information about the density, temperature, and velocity of the observed particles within the jet. An inhomogeneous broadening of the lines is thus produced when the expansion is optically probed in a direction perpendicular to the jet axis, where different groups of molecules contribute to the lineshape with a specific Doppler shift and a specific weight given by their number density. This link between the flow field of the jets and the lineshape of spectra collected from them has been analyzed by means of calculations [17], and experimentally [5, 18, 19].

In the present work, lineshape effects in spectra recorded by stimulated Raman spectroscopy (SRS) in axisymmetric supersonic jet expansions of nitrogen are investigated. This technique has been used with a great field depth, so it scans and integrates along a line of sight crossing the jet. We focus the attention on a region of the jet very close to the nozzle exit, where strong gradients of density and temperature occur and the experimental data can serve as a test for the usual jet models devised for farther regions. This is a region characterized by a high collision frequency, where three-body collisions are still present and different phenomena of great interest start, like nucleation of aggregates and the relaxation of internal degrees of freedom in molecular jets [1]. Furthermore, from a technological viewpoint, the experimental study of this region is very important. In fact, the nearnozzle flow field is difficult to model due to the interaction between the gas and the nozzle wall surface, and its simulations require detailed experimental measurements to anchor the calculations [20]. In spite of it, very few works have studied the spectral lineshape produced by the near-nozzle flow field [5], most of them analyzing jet regions far away from the nozzle $[18,19]$.

We report here experimental spectra, recorded from points very close to the nozzle exit, featuring a lineshape consisting of a splitting and an inhomogeneous broadening, which are mainly due to the strong density gradient 
in this region of the expansion and to different Doppler shifts. We have simulated the Raman spectra produced in the near-nozzle flow field obtained with simple models, usually applied far away from the nozzle, and compared them with the experimental spectra. This work allows testing the validity of these models in a region very close to the nozzle, and establishing some of their limitations.

A similar lineshape with a double-peak structure was reported previously in infrared absorption $[4,5,11]$ and FTMW $[8,21]$ measurements on supersonic expansions, and was attributed exclusively to the effect of strong condensation on the jet axis. The lineshape we present here has a different origin, though our simulation can reproduce the just mentioned results including some degree of condensation, as it will be shown. A correct interpretation of these spectroscopic measurements in the vicinity of the nozzle has to take into account both efects, the flow field gradients and the possible molecular aggregation.

In the paper, we briefly describe the experimental setup, to continue explaining the main aspects of the flow field model employed, and of the spectral lineshape produced by each flow element. Next, we discuss the influence of the flow model parameters on the final simulated Raman spectrum, and compare it with the experimental one, establishing limitations to the near-nozzle flow field. Finally, we introduce condensation into the model to analyze its effect on the spectral lineshape.

\section{EXPERIMENTAL}

The inverse Raman spectrometer at the IEM in Madrid was used for recording the spectra from the supersonic jets. It is based on the design by Owyoung [22] and its implementation at the IEM has been described previously [23], so only the most relevant details will be given here.

Nitrogen was expanded from a commercial solenoid driven pulsed valve. The source pressure, $P_{0}$, ranged from 1.5 to 3.5 bar. In all experiments the pulsed valve was kept at room temperature, $T_{0}=295 \mathrm{~K}$. The jet source was mounted on a movable base that could be displaced under vacuum. The movement along the jet axis was achieved by means of a linear motion feedthrough provided with a micrometric screw. Nearly square gas pulses of about $1 \mathrm{~ms}$ duration were used in the present experiments, and were expanded into a $50 \ell$ chamber pumped by a $2000 \ell \mathrm{s}^{-1}$ oil diffusion pump. The SRS measurements were performed on the central part of the gas pulses (quasy steady state), and special care was paid to the determination of the effective nozzle diameter $D_{\text {eff }}$ from measurements of the gas flow to the expansion chamber [24]. The $D_{\text {eff }}$ values were found to be $20-30 \%$ smaller than the nominal diameter of $0.5 \mathrm{~mm}$ and, from now on, we fix its value $D=0.4 \mathrm{~mm}$.

For the SRS measurements pump and probe laser beams were almost collinearly focused on the jet axis $z$, propagating along a direction $y$ not strictly perpendicular to it, see the bottom of Fig. 1. The laser beams formed
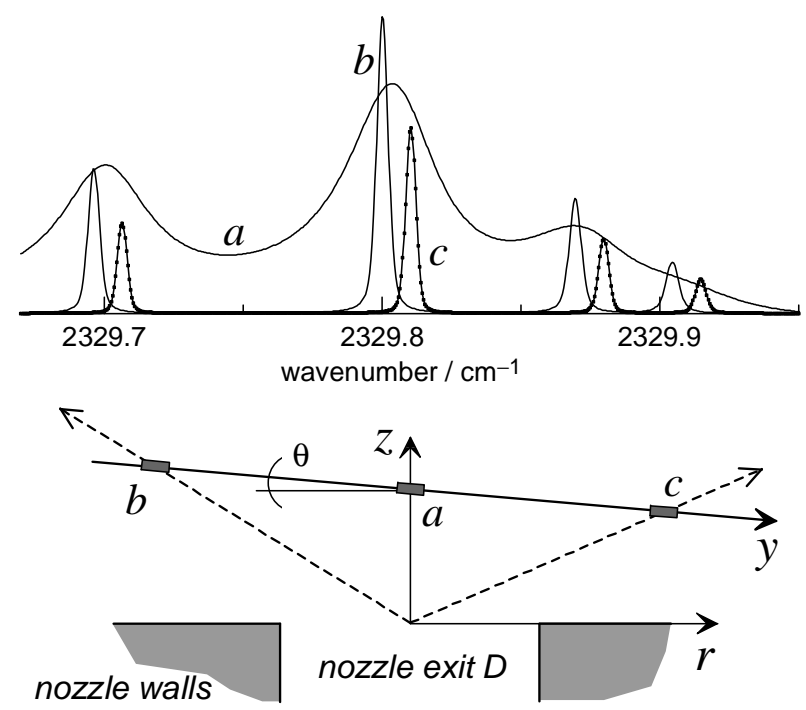

FIG. 1: Bottom: Geometry of the experimental set-up; $z$ is the jet axis; $y$ is the laser beam direction; dashed lines indicate the velocity vector field. Top: Raman spectra produced by the regions of the flow field marked as $a(z / D=0.5), b$ $(y / D=-1)$, and $c(y / D=+1)$, flow parameters are the same as in Fig. 6.

an angle of around $1^{\circ}$ in order to avoid the atmospherical nitrogen signal from the laser paths outside the chamber. The slight misalignment between the laser beams and the perpendicular direction $r$ to the jet axis, $\theta \approx 5^{\circ}$, allows a better assesment of the model employed in the simulation, as it yields an asymmetry in the lineshape of the spectra.

The probe beam was provided by a frequency stabilized $\mathrm{Ar}^{+}$laser operating in single mode at $514 \mathrm{~nm}$. For the pump beam, the emission of a ring dye laser was pulse amplified in a three stage amplifier, pumped by the second harmonic of a seeded Nd:YAG laser. The dyes used were Rhodamine $6 \mathrm{G}$ in the ring dye laser and Kiton red in the amplifier. The spectral resolution $\left(\sim 3.0 \times 10^{-3}\right.$ $\mathrm{cm}^{-1}$ ) is the convolution of the lineshapes of both lasers. The lasers formed a gaussian beam within the jet with a Rayleigh range of around $6 \mathrm{~mm}(15 D)$ and a beam waist of radius (half width at half maximum intensity) $\approx 20 \mu \mathrm{m}$ $(0.05 D)$.

\section{SIMULATION}

The simulation of a Raman spectrum recorded in the present experiment is obtained by dividing the laserbeam interaction region into small cylindrical cells, inside which the flow quantities are supossed homogeneous. To check the influence of the width of the cell, simulations have been carried out using for the laser beam cross section the true gaussian distribution of intensity. Taking into account the dimensions of the focussing, discussed 
above, the inhomogeneity of the flow in the cross section of the laser beam is small, and the effect of its radial intensity distribution on the simulation is negligible. Thus, the cell is only defined by its length that is fixed to $0.1 D$. To obtain the total spectrum, the signal of all cells is added. This addition is possible because the depletion of the probe laser intensity is extremely small, and deviations of the linearity are negligible for the densities of the jet.

These characteristics are in strong contrast to those of not tightly focussed absorption techniques, for which the interaction region is wider, the flow inhomogeneities along the cross section of the probe radiation affect the recorded spectrum, and the probe radiation intensity can vary in each point of the expansion [19].

There are two main aspects to consider in the simulation. The first one is a modelization of the flow field that gives the flow quantities at each cell, and the second one is a temperature-dependent model of the pressure broadening of the nitrogen $Q$-branch lines, and of their linemixing effects, to simulate the Raman spectrum of each cell. The combination of the steep gradients of density and temperature close to the nozzle, the divergent flow velocities, and the great variation of the homogeneous broadening along the laser beam, yields the splitting and inhomogeneous broadening observed in the experimental spectra.

As an example, three simulated spectra produced by three different cells distributed along the laser beam are shown in Fig. 1. The density and pressure in cell $a$ are high enough to produce a strong collisional broadening and line-mixing effect, as can be seen in the corresponding spectrum $a$. Meanwhile, cells $b$ and $c$ produce spectra with almost gaussian-like lines, just reflecting the convolution of the Doppler distribution and the spectral resolution, but with opposite Doppler shifts. When adding up the spectra of the cells along the $y$ direction to obtain the total spectrum at a given $z / D$, the global lineshape is an inhomogeneous combination of all the above effects.

\section{A. Flow Field}

To characterize the flow field it is necesary to give values of the density, the temperature, and the flow velocity in each point. Other quantities like the pressure are obtained assuming ideal gas behaviour. To model the flow field of the expansion we assume an isentropic and steady flow in the zone of silence, and cylindrical symmetry. The isentropic character of the flow is not true in other regions, like in the inner tube of the nozzle or in shock waves [25]. Though we are considering points in the jet very close to the nozzle we expect only small perturbations in this region, through a shock wave system, due to the interactions of the nozzle rim and the holder walls with the flow. As the analyzed expansions are pulsed and so the background pressure is maintained very low, these shock waves, as well as the barrel shock enclosing the zone of silence in continuous jets, are minimized and fade out very fast downstream.

The isentropic evolution of the density $n$ along the expansion axis $z$ is given by

$$
n\left(z, z_{0}\right)=n_{0}\left[1+0.5(\gamma-1) M\left(z, z_{0}\right)^{2}\right]^{-1 /(\gamma-1)},
$$

where $n_{0}$ is the stagnation density; the Mach number, $M$, of the flow is determined with the correlations given by [1] for an axisymmetric expansion with the specific heat ratio $\gamma=1.4$ for nitrogen, and $z_{0}$ is the origin of the expansion, which is defined as the point where $M=1$. For capillary nozzles it has been shown that this origin is inside the nozzle [26], and it will be fixed at $z_{0} / D=$ -0.4 in the simulations, by analogy with other analysis of continuous expansions of nitrogen through a capillary nozzle of $D \approx 0.31 \mathrm{~mm}$ [27]. The origin $z_{0}$ has a great influence in the values of the density very close to the nozzle, though after some distance downstream its effect in the flow quantities is negligible.

The density along a direction $r$ perpendicular to the axis $z$, has been modelled with the usual expresion [1]

$$
n(r, z)=n(0, z) \cos ^{2}(\alpha) \cos ^{2}(\phi \alpha),
$$

given by Ashkenas and Sherman [28], with $\alpha=$ $\arctan (r / z)$, and where $\phi$ is a parameter dependent on the specific heat ratio $\gamma$; for nitrogen $\phi=0.9451$. The formula (2) is a fit to data from method-of-characteristics calculations in the supersonic region, and is expected to be less reliable in the close vicinity of the nozzle, where the details of the transonic flow in the nozzle exit are still present. Measurements of the off-axis density in continuous expansions [27] show that the density falls off along a perpendicular direction to the axis faster than the predictions of Eqn (2). This effect can be modelled by modifying the parameter $\phi$. The strong influence of changing $\phi$ on the calculated shape of the absorption line across a free jet has been studied in [17].

The flow temperature is determined with the isentropic expresion

$$
T=T_{0}\left(n / n_{0}\right)^{\gamma-1},
$$

which gives the temperature at any point of the flow, knowing its density, and the stagnation values of density, and temperature $T_{0}$. For molecular gases a breakdown of the equilibrium between the rotational and translational degrees of freedom can occur in a supersonic expansion, yielding a rotational temperature higher than the translational one. In this work we assume equilibrium between both temperatures, because the jets studied are produced at high stagnation pressures that maintain this equilibrium until a large distance from the nozzle $[9,29]$. The off-axis rotational temperature has been proved [30] to follow the isentropic expression (3) along a perpendicular length of several $D$, before reaching the lateral shock wave which causes an increase in $T$. The lateral shock wave produces also an increase of the density, but at a larger distance from the axis than that at which the 


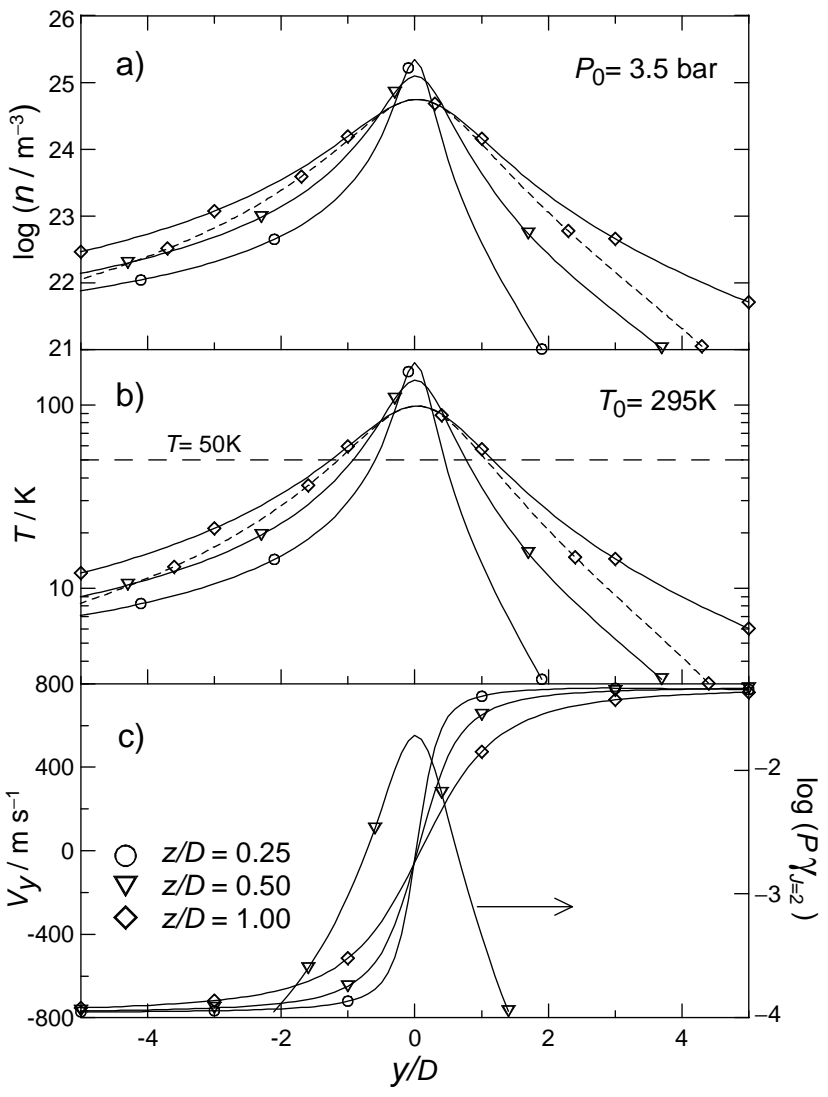

FIG. 2: Number density $n$ (molecules $\mathrm{m}^{-3}$ ) (a), temperature $T$ (b), and velocity component $V_{y}(\mathrm{c})$, along $y$ direction crossing the axis at three $z / D$ positions, forming an angle $\theta=5^{\circ}$ with the radial direction. In all the cases $P_{0}=3.5$ bar, $T_{0}=295 \mathrm{~K}, z_{0} / D=-0.4 ; \phi=0.9451$ (continuous lines), $\phi=1.05$ (dashed lines). The HWHM of the Lorentz contribution, $P \gamma_{J}\left(\mathrm{~cm}^{-1}\right)$, for the spectral line $Q(J=2)$ along one direction is shown in (c).

temperature rises, the same as in normal shock waves [31]. The influence of the lateral shock wave is stronger in regions close to the nozzle, and can limit the lowest temperature reached by the flow.

The absolute value of the flow velocity, $V$, at a given point is

$$
V=\left[\frac{2 \gamma k_{B}}{m(\gamma-1)}\left(T_{0}-T\right)\right]^{1 / 2},
$$

where $k_{B}$ is the Boltzmann constant and $m$ the molecular mass. The velocity field is modelled supossing that all the streamlines radiate from a point source located at the center of the exit plane of the nozzle. Actually, the streamlines just in the exit plane of the nozzle are almost parallel, diverging afterwards, and produce the effect of a virtual source at a certain distance downstream of the orifice, from which all the streamlines seem to radiate [28]. Although we have to simulate the velocity field so close to the nozzle to have into account this effect, we maintain the streamline source at the geometric center of the exit plane of the nozzle (see Fig. 1), for lack of a better model and because velocity measurements in a supersonic nitrogen jet [32] show that the velocity vector field does not depart much from that assumption.

To simulate the Raman spectra we calculate the values of density, temperature, pressure, and absolute velocity in each cell of the flow in interaction with the lasers, according to Eqs. (1)-(4). The laser beam direction $y$ forms an angle $\theta$ with the normal direction $r$ to the expansion axis $z$ (see Fig. 1). With this geometry the parallel component $V_{y}$ of the flow velocity $V$ (Eqn (4)) to the laser beam is calculated. The limits of the laser-flow interaction used to simulate the total Raman spectrum are established by the points in the laser direction at which the density has decreased at least four orders of magnitude.

Number density $n$, temperature $T$, and velocity component $V_{y}$, along $y$ direction crossing the expansion axis at different $z / D$ points, are shown in Fig. 2. Note that density drops off very fast, and that over a length of very few $D$ it falls several orders of magnitude. Furthermore, the effect of the parameter $\phi$ of Eqn (2) on density and temperature is shown in Fig. 2a and 2b, comparing their values for $\phi=0.9451$, the model value for $\gamma=1.4$, and for $\phi=1.05$.

\section{B. Spectral Lineshape}

The Raman spectrum corresponding to each point of the flow field is simulated with the molecular constants for ${ }^{14} \mathrm{~N}_{2}$ from reference [33]. The rovibrational frequencies $\omega_{J}$ are Doppler shifted according to the flow velocity component $V_{y}$ along the laser direction: $\omega_{J}=$ $\omega_{J, 0}\left(1+V_{y} / c\right)$. The width and lineshape of the Raman lines are obtained by the convolution of a Gaussian term, which is the result of the apparatus function and a Doppler broadening because of the local gas temperature, and a Lorentzian term due to a homogeneous collisional broadening associated to the local gas pressure.

The collisional broadening is simulated with the Lorenztian function $L_{J}(\omega)=\left(\Gamma_{J} / \pi\right) /\left[\left(\omega_{J}-\omega\right)^{2}+\Gamma_{J}^{2}\right]$, where $\Gamma_{J}=P \gamma_{J}$ is its half width at half maximum (HWHM); $P$ is the pressure, and $\gamma_{J}$ are coefficients that depend on the rotational number $J$ and the temperature. The dependence of these coefficients on temperature is very strong for $T<200 \mathrm{~K}$, and it has been modelled with the expresion [34]

$$
\gamma_{J}(T)=\gamma_{J, 0}\left(\frac{T_{0}}{T}\right)^{m_{J}},
$$

fitted to the experimental values of $\gamma_{J}$ given in reference [35]. The evolution of $\gamma_{J}(T)$ for the different $J$ values and the corresponding fitted parameters are shown in Fig. 3. The strong variation of pressure in the flow very close to the nozzle exit produces a very broad Lorentzian component on the jet axis, decreasing rapidly outwards 


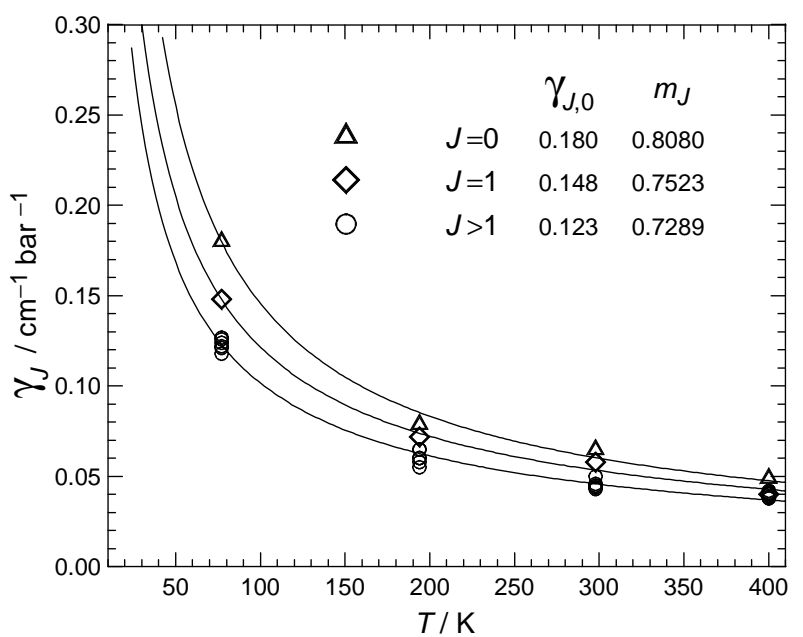

FIG. 3: $Q$-branch line broadening coefficients $\gamma_{J}$ of $\mathrm{N}_{2}$ taken from Ref. [35] (symbols), and the fits of Eqn (5) to them (continuous lines), with their fitted parameters tabulated inside.

from the axis, as can be seen in Fig. 2c for the spectral line $Q(2)$. In this way, the spectral lines from the axial points of the jet are broader and have higher wings than the lines from molecules outwards from the expansion core, as it is shown in Fig. 1.

Line mixing effects could also be relevant for high pressure values close to the nozzle exit. In fact, the largest pressure sampled in the experiments corresponds to the axial point at $z / D=0.25$, where $P \approx 0.5$ bar. However, this effect becomes negligible very fast with growing distance to the nozzle, and is only significant for the spectrum at $z / D=0.25$ of the expansion at $P_{0}=3.5$ bar.

The collisional line mixing effect is included in the intensity profile $I_{J}(\omega)$ by a perturbative treatment, which leads to a mixing term added to the Lorentzian contibution [36]. For the $J$ line the final expresion is

$$
I_{J}(\omega) \propto \frac{\Gamma_{J} / \pi+\left(\omega-\omega_{J}\right) Y_{J}}{\left(\omega-\omega_{J}\right)^{2}+\Gamma_{J}},
$$

where $Y_{J}$ is the mixing coefficient. The mixing coefficients $Y_{J}$ for the nitrogen $Q$-branch have been taken from [37], where they are given at $298 \mathrm{~K}$.

\section{RESULTS AND DISCUSSION}

High resolution Raman spectra of the $Q$ branch of the fundamental vibrational mode of $\mathrm{N}_{2}$ between 2329 and $2330 \mathrm{~cm}^{-1}$ were recorded from different expansions of nitrogen, the lasers crossing the jet axis in the range $z / D=0.25-12.5$. Some of the recorded Raman spectra are shown in Fig. 4. Two main features appear in these spectra, an asymmetric splitting of the lines, and a strong Lorentzian character, both more pronounced at

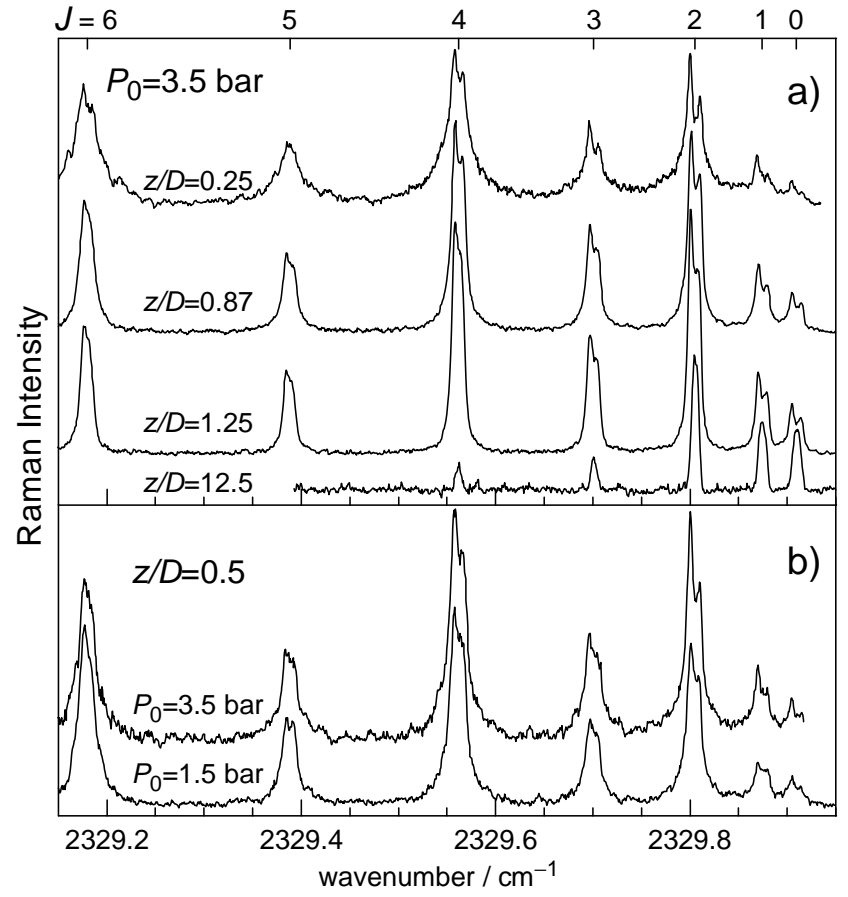

FIG. 4: Experimental Raman spectra of the $Q$ branch of $\mathrm{N}_{2}$ expanded at $P_{0}=3.5$ bar at several axial positions $z / D$ (a), and at $z / D=0.5$ with two stagnation pressures (b).

higher pressures, and at distances closer to the nozzle. At distances over $z / D=10$ these features have completely disappeared (see spectrum at $z / D=12.5$ in Fig. 4a).

Several examples of the simulated spectra for different flow field and stagnation conditions are shown in Fig. 5, to illustrate the effect on the total spectrum of the stagnation pressure, a temperature constraint, and the off-axis density model. Two spectra with stagnation pressures $P_{0}=1.5$ and 3.5 bar are represented in Fig. $5 \mathrm{a}$, showing that the dip in the lineshape is deeper for the higher pressure, and that the wings of the lines are steeper for the lower pressure. Both features are in accordance with the observations (see Fig. 4b). Furthermore, these simulated spectra are obtained with the laser beam strictly orthogonal to the expansion axis, so the asymmetry in the double-peak structure does not appear. The effect of limiting the minimum flow temperature is shown in Fig. 5b, where two simulated spectra are plotted, one of them with no constraint on the temperature, so with minimum temperature $T_{\min }=0 \mathrm{~K}$, and the other one with $T_{\min }=50 \mathrm{~K}$. In regions so close to the nozzle exit, the flow can be perturbed by the nozzle and its holder through a system of lateral shock waves, therefore the expanded gas does not reach temperatures so low as those in a free jet. Figure $5 \mathrm{c}$ shows the dependence of the simulated spectra on the off-axis density model, Eqn (2), modified by variations of the parameter $\phi$. We have taken two extreme values of $\phi$ to show the differences, with $\phi=0$ the density falls much slower along the radial 


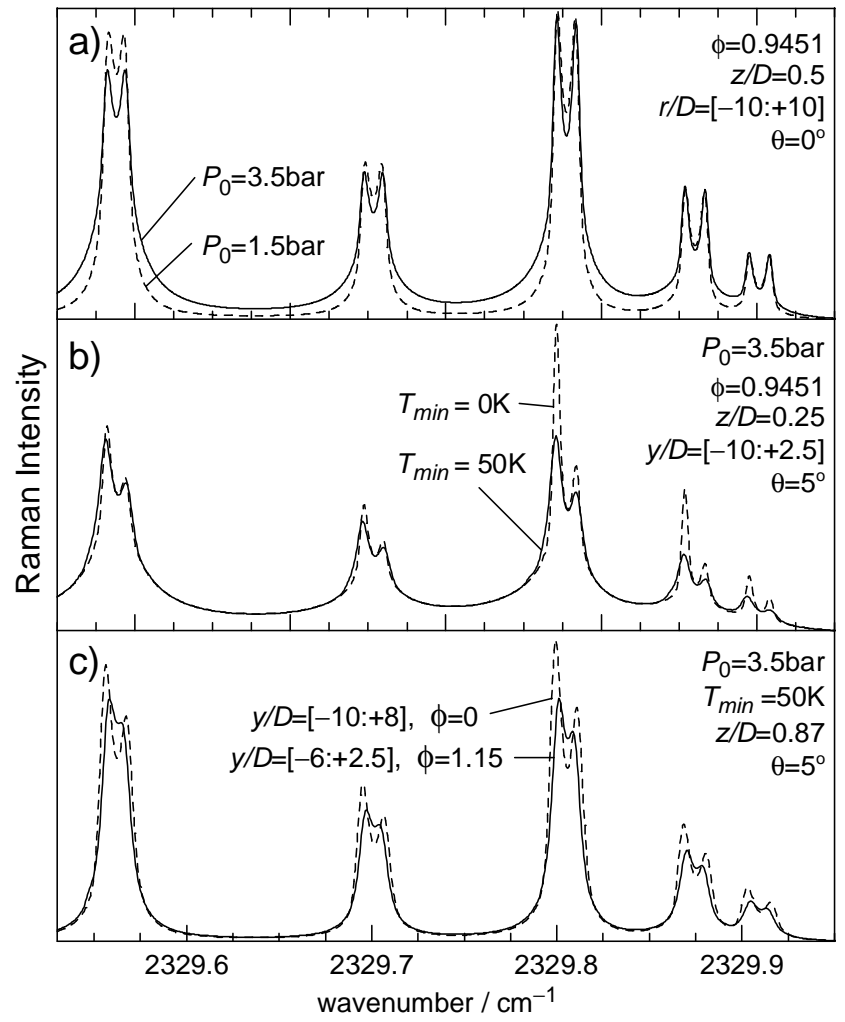

FIG. 5: Simulations of $Q$-branch Raman spectra. (a) Two expansions with different $P_{0}$, with no constraint on the temperatures. (b) An expansion with and without a limit in the minimum temperature, $T_{\min }$. (c) An expansion with two different values of $\phi$ for the radial density model, Eqn (2). In all the simulations $T_{0}=295 \mathrm{~K}$, and $z_{0} / D=-0.4$.

direction than with $\phi=1.15$ (see Fig. 2a). In the calculated spectra, the double-peak structure in the lineshape is more pronounced and the dips deeper when the density falls slowly. On the contrary, the peaks almost disappear for a faster off-axis density drop, though the line width does not change.

The good agreement between the experimental and the simulated spectra obtained by the present model is shown in Fig. 6. The experimental stagnation values of pressure and temperature were fixed, and the sonic point located at $z_{0} / D=-0.4$, as discussed above. The lineshape asymmetry led to assume a laser beam inclination angle, which was establised at $\theta=5^{\circ} \pm 1^{\circ}$. In the usual model of density and temperature of the flow, the parameter $\phi$ of Eqn 2, and the minimum temperature of the flow, $T_{\text {min }}$, had to be modified to account for the experimental data. Their final values and the physical implications are described in the following.

Firstly, to obtain a better agreement in the relative intensities of lower $J$, the model was modified setting a minimum temperature in the flow (see Fig. 5b), with the final value of $T_{\min }=50 \pm 10 \mathrm{~K}$. Measurements of rotational temperature in continuous expansions of $\mathrm{N}_{2}$ along

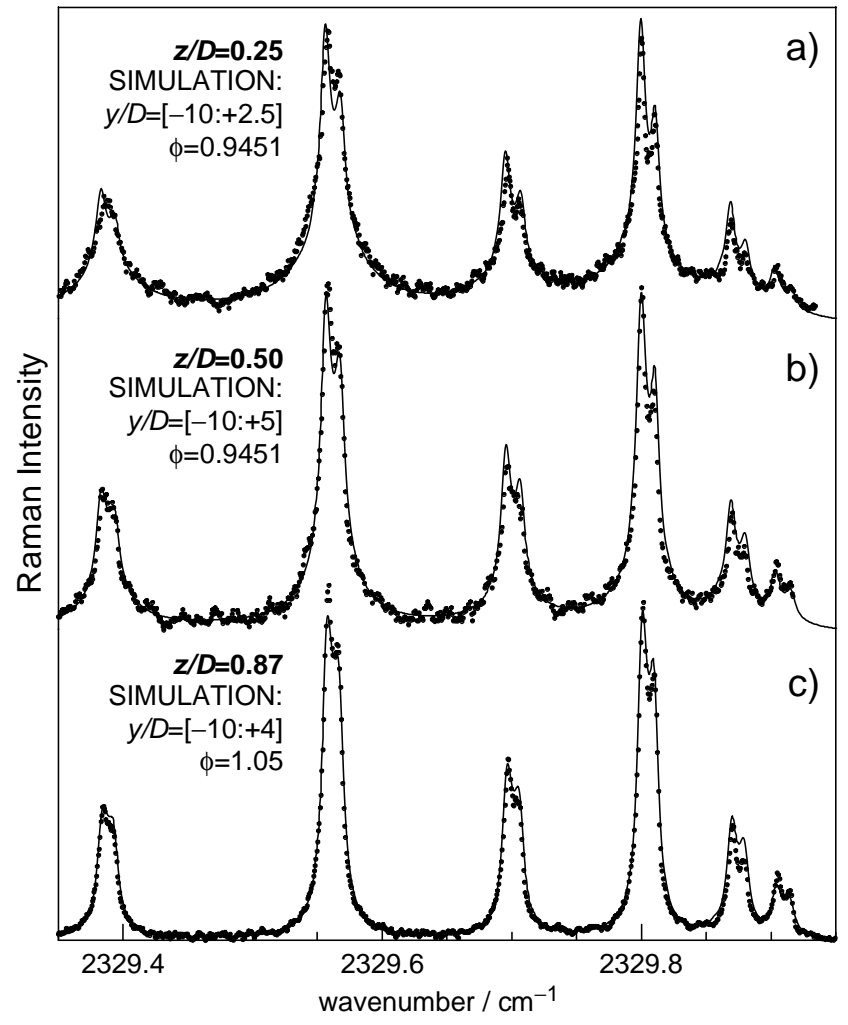

FIG. 6: Comparison of some experimental spectra (solid circles) for $P_{0}=3.5 \mathrm{bar}$, with the simulated spectra (lines). The common parameters for all the simulations are: $T_{0}=295 \mathrm{~K}$, $z_{0} / D=-0.4, \theta=5^{\circ}$, and $T_{\min }=50 \mathrm{~K}$.

the $r$ direction at $z / D=2[30]$, have shown that the temperatures do not decrease as much as the model predicts, due to the influence of the shock barrel, obtaining a minimum rotational temperature of around $30 \mathrm{~K}$. In the present work, the perturbance by lateral shock waves and the nozzle is expected to be larger, because the flow is very close to the nozzle orifice.

The second modification was introduced to fit the relative intensities of the double peak structure of the spectral lines at $z / D>0.5$. It consists of setting a value of the parameter $\phi$ larger than the given value [28] for nitrogen $(\phi=0.9451)$, which reduces the depth of the line dips (see Fig. 5c). This change of $\phi$ means that the off-axis density drops radially faster than the model predictions for the nitrogen $\gamma=1.4$ (see Fig. 2a), a fact already observed in radial density measurements in continuous expansions of $\mathrm{CO}_{2}$ [27]. A comparation between experiment and simulation leads to an increasing value of $\phi$, which ranges from $\phi=0.9451$ for the spectra at $z / D \leq 0.5$, Fig. $6 \mathrm{a}$ and b, to $\phi=1.05$ for the spectrum at $z / D=0.87$, Fig. $6 c$, and $\phi=1.15$ for a farther spectrum at $z / D=1.25$; all these values have a sensibility of about $\pm 10 \%$. It can be concluded that the radial decrease in density becomes faster with growing distance to the nozzle. 


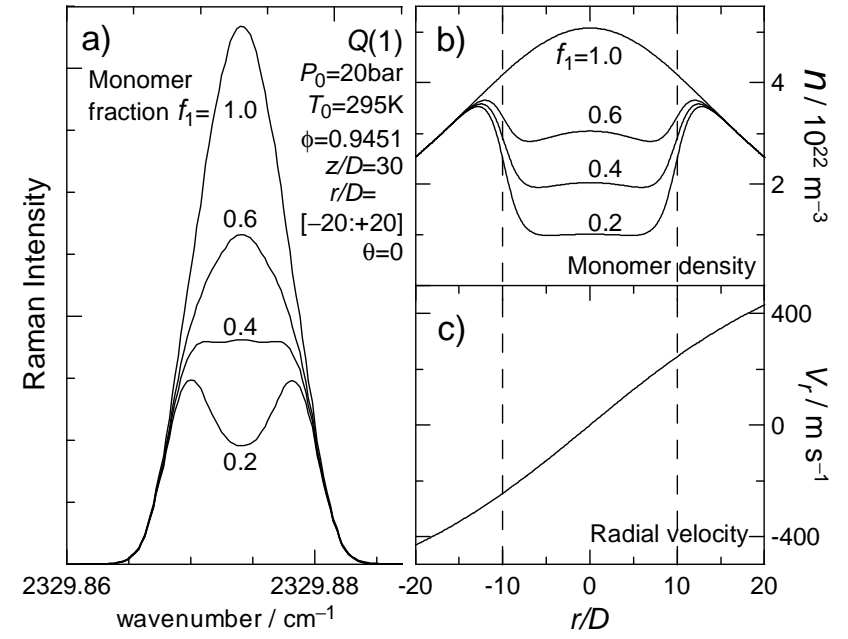

FIG. 7: Effect on the line-shape of the condensation of the expanded gas in the core of the jet, specified with the fraction $f_{1}$ of molecules that has not aggregated. (a) Simulated $Q(1)$ line. (b) The density of monomers along the radial direction that generates the spectra. (c) Radial velocity of the monomers. The vertical dashed lines indicate the radius that limits the region where condensation is present.

A lineshape with a double peak structure has been also reported in works on infrared absorption spectroscopy in supersonic expansions. It was firstly observed in $\mathrm{NH}_{3}$ expansions [4], described as a clear deviation of the gaussian form, with a splitting that appears and becomes more pronounced with increasing stagnation pressure. This line profile was finally attributed [5] to clustering that is concentrated on the axis and depletes the proportion of monomers with Doppler shift around zero, yielding a dip in the absorption profile. This type of infrared absorption lineshape has been also observed in pulsed expansions of mixtures of $\mathrm{CO}_{2}$ with rare gas [38], in continuous expansions of methane [6], though no absorption features attributable to methane clusters could be found by the authors of this last work, and in continuous expansions of acetylene [11]. Others works using Fourier transform microwave spectroscopy on pulsed expansions of OCS and rare gas complexes of OCS have described a Doppler splitting of rotational lines [39], concluding that much of this doubling effect is based on an axial depletion of the monomer in the core of the jet due to polimerization [8]. The same lineshape was reported in FT microwave spectroscopy of pulsed jets of mixtures of a rare gas and fluorobenzene [21]. In most of these works the measurements were carried out far away from the nozzle exit, except in $\mathrm{NH}_{3}$ expansions [5], in which spectra were recorded from $z / D=0.6$. The lineshape observed in all these works is explained mainly by condensation, as a combination of the Doppler shift from molecules moving along different streamlines, and the monomer depletion on the jet axis caused by clustering, with no discussion about the effect of the near-nozzle flow field.
The lineshape we report here is not attributable to condensation. First of all, the double peak structure shown in Fig. 4 disappears from the spectrum with growing distance to the nozzle, while the phenomenon of aggregation is such that the fraction of free monomers in the jet axis decreases with distance [40], and so the dip in the lineshape should get deeper instead. Moreover, studies of condensation in continuous expansions of $\mathrm{N}_{2}$ through a nozzle of $D=0.31 \mathrm{~mm}$ [27] show that nitrogen starts to aggregate at stagnation pressures of around 4 bar, but at distances very far from the nozzle, at $z / D \approx 10$. These results rule out the occurrence of condensation in our experiments.

We have used the present model to simulate the lineshape expected in a hypothetic jet of nitrogen with condensation, in order to reproduce the lineshape reported in the expansions with condensation discussed above. An expansion at $P_{0}=20$ bar and $T_{0}=295 \mathrm{~K}$ was chosen, with the laser beam crossing the jet axis at $z / D=30$, and with different monomer fractions $f_{1}$, indicative of the condensation degree in the expansion. Experimental data of monomer fractions in continuous expansions of $\mathrm{CO}_{2}$ [40] have values almost constant in a wide region around the axis jet, so we have taken constant values of $f_{1}$, ranging from 0.2 to 0.6 , in a core centered on the axis, with no condensation outside. The monomer fraction changes smoothly across the border of the core, placed at $r / D=10$, until condensation vanishes completely (see Fig. 7b). The $Q(J=1)$ lineshape produced under these conditions in the Raman spectra is shown in Fig. 7a for different degrees of aggregation. As the monomer fraction decreases, the lineshape begins to deviate from the gaussian form, getting flatter on top and then splitting and showing a double-peak structure. A similar trend in the lineshape evolution was obtained in $\mathrm{NH}_{3}$ expansions with increasing stagnation pressures $[4,5]$, which is equivalent to increasing the aggregation. Note that the flanks of the profile simulated in Fig. 7 are steeper than those of the experimental spectra recorded close to the nozzle, which is indicative of the different physical origin of the lineshapes, with the broad wings in the present spectra pointing to collisional effects.

\section{CONCLUSIONS}

Stimulated Raman spectra have been recorded in pulsed axisymmetric expansions of nitrogen, scanning the near-nozzle flow field. A double-peak lineshape in the Raman spectra has been described, which is the result of the inhomogeneous broadening produced along the strong gradients of density and temperature that occur close to the nozzle exit.

A simulation of the Raman spectra obtained in the jets has been carried out, testing the validity of flow field models in the near-nozzle region. In the simulation we have employed the most usual flow field model [1, 28], plus spectroscopical parameters from various works [33, 
35, 37], and have obtained a good agreement with the experimental spectra, after some modifications referred to the flow.

Two aspects of the usual flow field model must be modified in order to account for the present observations on the vecinity of the nozzle. In the first place, a lower limit to the flow temperature must be introduced. This limiting lower temperature is likely due to the non-negligible interaction of the jet with the surroundings. In addition, a faster off-axis density drop than that predicted by the model has to be assumed, which becomes more pronounced with increasing axial distance to the nozzle.

A similar spectral lineshape, previously reported in absorption measurements in supersonic jets, was exclusively attributed to the depletion of monomers from the jet axis due to clustering. This lineshape and the one here reported are similar at first sight but have different physical origins. The simulation here carried out can also reproduce the former lineshape when condensation is present in the jet and is concentrated on the axis. However, with the present results, we can conclude that lineshape simulations close to the nozzle exit of expansions with con- densation have to include the effect of the flow field as described in this work, together with the depletion of monomers due to aggregation.

Summarily, we have shown how experimental lineshapes recorded from the near-nozzle flow field, like those reported in this work, are valuable as a test for the flow models in this region, where accurate modelling is difficult because of the interaction of the gas with the nozzle walls.

\section{ACKNOWLEDGMENTS}

A. Ramos acknowledges CSIC for an I3P grant. The technical advice of J M Castillo, M A Moreno and J Rodriguez has been most valuable for the achievement of the present results. This work has been funded by the DGI (MEC) of Spain under grants ENE 2006-14577C04-03/FTN, FIS 2004-02558, FIS 2005-02029, and FIS 2007-61686.
[1] D. R. Miller in Atomic and Molecular Beam Methods, ed. G. Scoles, Vol. I; Oxford University Press, 1988, chapter 2.

[2] S. DePaul, D. Pullman, B. Friedrich, J. Phys. Chem. 1993, 97, 2167.

[3] B. S. Zhao, M. Castillejo, D. S. Chung, B. Friedrich, D. Herschbach, Rev. Sci. Instrum. 2004, 75, 146.

[4] Y. Mizugai, H. Kuze, H. Jones, M. Takami, Appl. Phys. B 1983, 32, 43.

[5] K. Veeken, J. Reuss, Appl. Phys. B 1984, 34, 149.

[6] A. Amrein, M. Quack, U. Schmitt, J. Phys. Chem. 1988, 92, 5455 .

[7] M. Herman, R. Georges, M. Hepp, D. Hurtmans, Int. Rev. Phys. Chem. 2000, 19, 277.

[8] F. J. Lovas, R. D. Suenram, J. Chem. Phys. 1987, 87, 2010.

[9] F. J. Aoiz, T. Díez-Rojo, V. J. Herrero, B. MartínezHaya, M. Menéndez, P. Quintana, L. Ramonat, I. Tanarro, E. Verdasco, J. Phys. Chem. A 1999, 103, 823.

[10] G. A. Amaral, F. J. Aoiz, L. Bañares, J. Barr, V. J. Herrero, B. Martínez-Haya, M. Menéndez, G. A. Pino, I. Tanarro, I. Torres, J. E. Verdasco, J. Phys. Chem. A 2005, 109, 9402.

[11] K. Didriche, C. Lauzin, P. Macko, W. J. Lafferty, R. J. Saykally, M. Herman, Chem. Phys. Lett. 2008, 463, 345.

[12] P. M. Felker, P. M. Maxton, M. W. Schaeffer, Chem. Rev. 1994, 94, 1787.

[13] L. Abad, D. Bermejo, V. J. Herrero, J. Santos, I. Tanarro, J. Phys. Chem. A 1997, 101, 9276.

[14] M. J. Bass, M. Brouard, R. Cireasa, A. P. Clark, C. Vallance, J. Chem. Phys. 2005, 123, 094301.

[15] M. Brouard, R. Cireasa, A. P. Clark, G. C. Groenenboom, G. Hancock, S. J. Horrocks, F. Quadrini, G. A. D. Ritchie, C. Vallance, J. Chem. Phys. 2006, 125, 133308.
[16] V. Poterya, O. Votava, M. Fárník, M. Ončák, P. Slavíček, U. Buck, B. Friedrich, J. Chem. Phys. 2008, 128, 104313.

[17] M. A. Gaveau, D. Boscher, J. P. Martin, Chem. Phys. Lett. 1984, 10\%, 31.

[18] K. Didriche, P. Macko, M. Herman, J. Thiévin, A. Benidar, R. Georges, JQSRT 2007, 105, 128.

[19] E. Pawelec, V. Caubet-Hilloutou, S. Mazouffre, Plasma Sources Sci. Technol. 2007, 16, 635.

[20] I. D. Boyd, D. B. VanGilder, E. J. Beiting, AIAA J. 1996, 34, 2320.

[21] M. Schäfer, A. Bauder, Chem. Phys. Lett. 1999, 308, 355.

[22] R. S. McDowell, C. W. Patterson, A. Owyoung, J. Chem. Phys. 1980, 72, 1071.

[23] J. Santos, P. Cancio, J. L. Doménech, J. Rodríguez, D. Bermejo, Laser Chem. 1992, 12, 53.

[24] L. Abad, D. Bermejo, V. J. Herrero, J. Santos, I. Tanarro, Rev. Sci. Instrum. 1995, 66, 3826.

[25] M. J. Zucrow, J. D. Hoffman, Gas Dynamics, Vol. I, Wiley, New York, 1976.

[26] H. R. Murphy, D. R. Miller, J. Phys. Chem. 1984, 88, 4474.

[27] A. Ramos, Procesos de intercambio energetico en expansiones supersonicas: Estudio por espectroscopia Raman, PhD Thesis, Universidad Complutense, Madrid, 2001.

[28] H. Ashkenas, F. S. Sherman in 4th Rarefied Gas Dynam$i c s$, ed. J. H. de Leeuw, Vol. II; Academic Press, New York, 1966, pp. 84.

[29] A. Ramos, G. Tejeda, J. M. Fernández, S. Montero, Phys. Rev. A 2002, 66, 022702 .

[30] A. Ramos, G. Tejeda, J. M. Fernández, S. Montero, J. Phys. Chem. A 2009, 113, 8506.

[31] A. Ramos, B. Maté, G. Tejeda, J. M. Fernández, S. Montero, Phys. Rev. E 2000, 62, 4940.

[32] H. Moosmüller, G. C. Herring, C. Y. She, Opt. Lett. 
1984, 9, 536 .

[33] J. Bendtsen, F. Rasmussen, J. Raman Spectrosc. 2000, 31, 433.

[34] G. C. Herring, B. W. South, JQSRT 1994, 52, 835.

[35] L. Gómez, Parametros de ensanchamiento colisional de moleculas de interes atmosferico por espectroscopia de perdida Raman estimulada, $\mathrm{PhD}$ Thesis, Universidad Complutense, Madrid, and Université de Franche Comté, France, 2007.

[36] A. Lévy, N. Lacome in Spectroscopy of the Earth's Atmosphere and interstellar medium, ed. K. N. Rao and
A. Weber; Academic Press Inc., 1992.

[37] G. J. Rosasco, W. Lempert, W. S. Hurst, A. Fein, Chem. Phys. Lett. 1983, 97, 435.

[38] R. W. Randall, M. A. Walsh, B. J. Howard, Faraday Discuss. Chem. Soc. 1988, 85, 13.

[39] E. J. Campbell, L. W. Buxton, T. J. Balle, M. R. Keenan, W. H. Flygare J. Chem. Phys., 1981, 74, 829 .

[40] A. Ramos, J. M. Fernández, G. Tejeda, S. Montero, Phys. Rev. A 2005, 72, 053204. 